\title{
Phenoscape: Semantic analysis of organismal traits and genes yields insights in evolutionary biology
}

\author{
Paula M. Mabee ${ }^{1}$, Wasila M. Dahdul ${ }^{1}$, James P. Balhoff ${ }^{2}$, Hilmar Lapp ${ }^{3}$, Prashanti Manda ${ }^{4}$, \\ Josef Uyeda $^{5}$, Todd Vision ${ }^{6}$, Monte Westerfield ${ }^{7}$ \\ ${ }^{1}$ Department of Biology, University of South Dakota, Vermillion, South Dakota, USA \\ ${ }^{2}$ Renaissance Computing Institute, University of North Carolina, Chapel Hill, North Carolina, \\ USA \\ ${ }^{3}$ Center for Genomic and Computational Biology, Duke University, Durham, North Carolina, \\ USA \\ ${ }^{4}$ Department of Computer Science, University of North Carolina at Greensboro, North Carolina, \\ USA \\ ${ }^{5}$ Department of Biological Sciences, Virginia Tech, Blacksburg, Virginia, USA \\ ${ }^{6}$ Department of Biology, University of North Carolina at Chapel Hill, North Carolina, USA \\ ${ }^{7}$ Institute of Neuroscience, University of Oregon, Eugene, Oregon, USA \\ Corresponding Author: \\ Paula M. Mabee ${ }^{1}$ \\ Email address: paula.mabee@usd.edu
}




\section{ABSTRACT}

2 The study of how the observable features of organisms, i.e., their phenotypes, result from the

3 complex interplay between genetics, development, and the environment, is central to much

4 research in biology. The varied language used in the description of phenotypes, however,

5 impedes the large scale and interdisciplinary analysis of phenotypes by computational methods.

6 The Phenoscape project (www.phenoscape.org) has developed semantic annotation tools and a

7 gene-phenotype knowledgebase, the Phenoscape KB, that uses machine reasoning to connect

8 evolutionary phenotypes from the comparative literature to mutant phenotypes from model

9 organisms. The semantically annotated data enables the linking of novel species phenotypes with

10 candidate genes that may underlie them. Semantic annotation of evolutionary phenotypes further

11 enables previously difficult or novel analyses of comparative anatomy and evolution. These

12 include generating large, synthetic character matrices of presence/absence phenotypes based on

13 inference, and searching for taxa and genes with similar variation profiles using semantic

14 similarity. Phenoscape is further extending these tools to enable users to automatically generate

15 synthetic supermatrices for diverse character types, and use the domain know ledge encoded in

16 ontologies for evolutionary trait analysis. Curating the annotated phenotypes necessary for this

17 research requires significant human curator effort, although semi-automated natural language

18 processing tools promise to expedite the curation of free text. As semantic tools and methods are

19 developed for the biodiversity sciences, new insights from the increasingly connected stores of

20 interoperable phenotypic and genetic data are anticipated. 
There are over 20 million extant species on the planet, most of which can be described in relation to their unique and widely diverse phenotypes. Comparisons across species phenotypes,

24 however, cannot yet readily be made using computer-assisted methods. This is because the rich

25 legacy of comparative morphology has not yet been semantically enabled - that is, the corpus is

26 in a free-text format that renders computation nearly impossible. This situation began to change

27 almost two decades ago when model organism geneticists began representing the phenotypic

28 changes resulting from experimental gene manipulations, with terms from anatomy or phenotype

29 ontologies that they developed for each model organism (e.g., Sprague et al. 2001). More

30 recently, the opportunity to enable interoperability from the phenotypes of biodiverse species to

31 candidate genes from model species (Mabee et al. 2007a, 2007b) motivated the Phenoscape team

32 to develop one of the first multispecies anatomy ontologies, the Teleost Anatomy Ontology

33 (Dahdul et al. 2010b), based initially on the Zebrafish Anatomy Ontology (Ruzicka et al. 2015).

34 Developing ontologies appropriate for biodiversity, including taxonomy ontologies (Midford et

35 al. 2013) and scaling them up first to the level of teleost fishes (Dahdul et al. 2010), then to the

36 level of vertebrates (Dahdul et al. 2012) and then to the level of metazoans (Mungall et al. 2012;

37 Haendel et al. 2014), further enabled the automation of phenotypic comparisons across vertebrate

38 species and discovery of candidate genes underlying evolutionarily novel phenotypes by the

39 team (Edmunds et al. 2016). Over the past ten years a broad community of scientists invested in

40 the development of shared community ontologies (e.g., Gkoutos et al. 2005; Haendel et al. 2008,

41 2014; Dahdul et al. 2014), annotation tools (Balhoff et al. 2010, 2014a; Yoder et al. 2010; Cui et

42 al. 2016; The Gene Ontology Consortium 2017) and formats (Dahdul et al. 2010a; Vos et al.

43 2012) for phenotype annotation across biodiverse species (Dahdul et al. 2010a). These resources 
44 have made computational analyses possible and they have been leveraged to build a wealth of

45 innovative applications (e.g., Deans et al. 2012; Mullins et al. 2012; Balhoff et al. 2013;

46 Dececchi et al. 2015; Manda et al. 2015; Druzinsky et al. 2016; Jackson et al. 2018) across a

47 variety of biodiversity-based research. The Phenoscape Knowledgebase (KB) (Figure 1)

48 demonstrates these connections by integrating gene phenotype annotations from model organism

49 databases with phenotype annotations from the biodiversity literature (Table 1). Compelling

50 demonstrations of the utility of semantics for biodiversity studies are important because of the

51 large and expensive investments in infrastructure and tool development required to curate the

52 legacy literature and move the publication of phenotypic data into a natively semantic form.

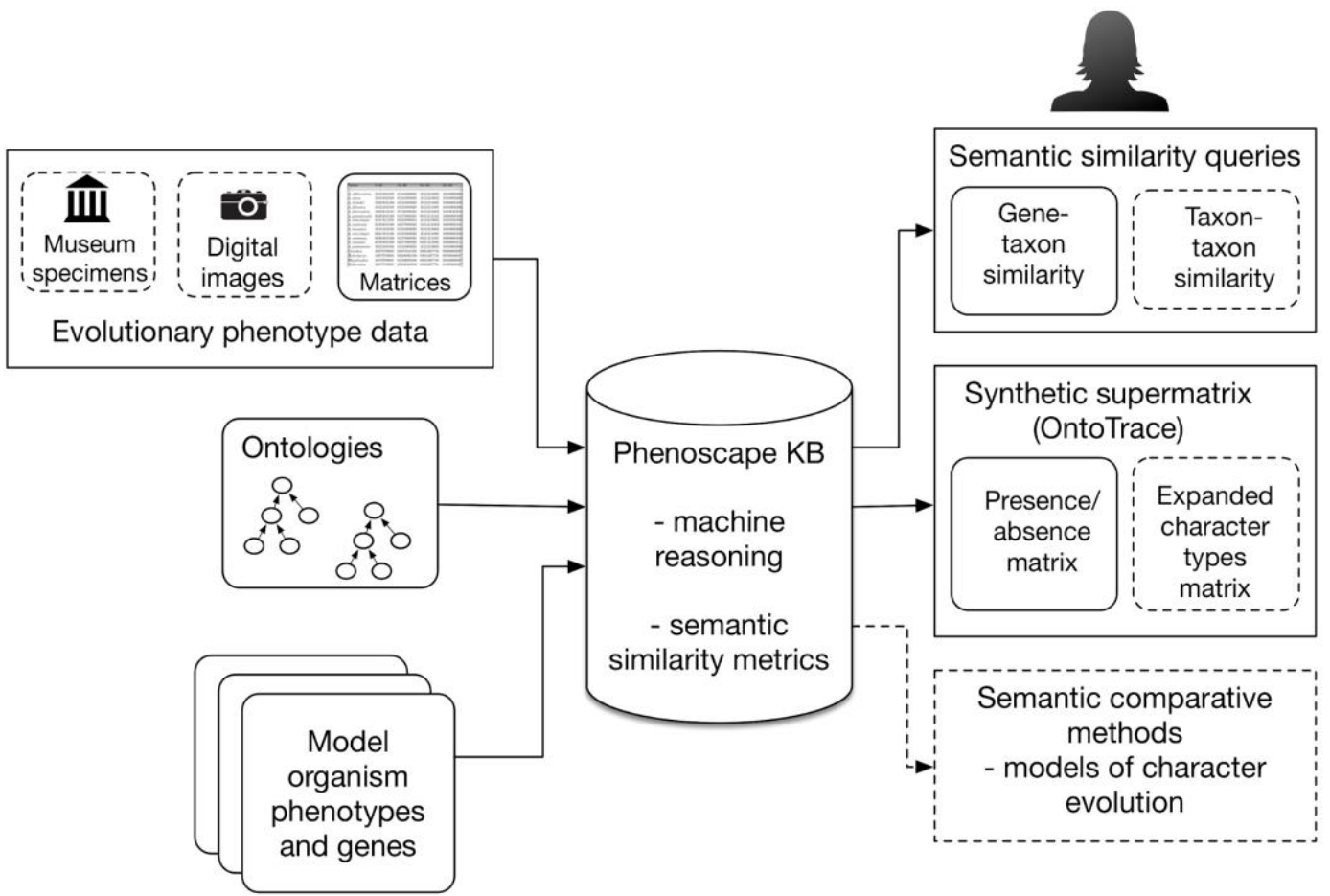

Figure 1. Flow chart of currently existing data sources and tools (solid borders and lines) in the

55 Phenoscape KB, and data and tools not yet integrated or developed (dotted borders and lines) but relevant to users in biodiversity research. 
58 Table 1: Data for evolutionary and model organism phenotypes in the Phenoscape KB. (Data as

59 of 2018-05-11)

60 Evolutionary Phenotypes

\begin{tabular}{|l|l|}
\hline Annotated anatomical character states & 22,321 \\
\hline Total number of annotated taxa (extant and fossil vertebrates) & 5,310 \\
\hline Total number of taxon phenotypes & 540,163 \\
\hline Terminal taxa (species) with at least one phenotype & 4,260 \\
\hline Non-terminal taxa with at least one phenotype & 1,050 \\
\hline Evolutionary phenotype profiles & 682 \\
\hline
\end{tabular}

61

62 Model Organism Phenotypes

\begin{tabular}{|l|c|c|c|c|}
\hline & Zebrafish & Mouse & Xenopus & Human \\
\hline phenotype & 5,883 & 7,758 & 12 & 3,717 \\
\hline Phenotype annotations & 90,132 & 171,876 & 236 & 123,956 \\
\hline Genes with any expression & 12,509 & 10,599 & 15,062 & 0 \\
data & & & & \\
\hline Gene expression & 179,232 & 800,824 & 454,337 & 0 \\
\hline
\end{tabular}


To date, only a small proportion of the biodiversity literature has been annotated

64 semantically, and no publisher, to our knowledge, tags phenotypes with ontological terms that

65 would support interoperability. The comparative study of organismal phenotypes, however,

66 motivates research across diverse fields of biology, including evolution, paleontology,

67 developmental biology, agriculture, and the veterinary and health sciences (Deans et al. 2015).

68 The efficiency and potential of fundamental discoveries in the biodiversity arena would be

69 dramatically expanded by the increased use of semantics. Further, few species, i.e., only model

70 organisms, have curated phenotypic data that is linked to genetic and genomic data. The growth

71 in sequencing technology, however, is changing this dynamic, resulting in the rapid expansion of

72 genomic data for non-model species (e.g., Russell et al. 2017 and Chapter 10). However, without

73 corresponding phenomic databases, the challenge of relating the growing volume of genetic

74 knowledge in model and emerging model organisms to the diversity of phenotypes in nature

75 cannot be met. In this chapter, through the description of driving research questions and by

76 examples of the use of semantically annotated data in the Phenoscape KB, we provide a glimpse

77 of the promise that semantic analysis tools hold in comparing phenotypes across species and

78 globally associating genetic to phenotypic data.

\section{Relating biodiverse phenotypes to candidate genes}

Identifying the genetic and developmental changes that brought forth the incredible

81 phenotypic diversification of life is a recalcitrant problem, but one where a basic semantic

82 approach has shown promise and where more sophisticated approaches using semantic similarity

83 may yet be even more valuable. Semantic similarity enables comparison and analysis of semantic

84 annotations between entities (genes, taxa) using ontologies and computational reasoners to

85 compute scores that reflect the level of similarity (e.g., Washington et al. 2009; Manda et al. 
2015; see examples in Chapter 10). The Phenoscape team showed that ontology-driven

87 information systems can generate thousands of testable hypotheses relating unique morphologies

88 from non-model biodiverse species to candidate genes (Mabee et al. 2012). One of these, for

89 example, connected the unique loss of a tongue ('basihyal element') in catfishes (Siluriformes)

90 with several candidate genes from the zebrafish data. Edmunds et al. (2016) experimentally

91 tested the candidates by examining their endogenous expression patterns in the channel catfish,

92 Ictalurus punctatus, and found results consistent with the in silico hypothesis that the tongue

93 evolved through disruption in developmental pathways at, or upstream of, brpfl.

94 The Phenoscape team recently extended this approach (Manda et al. 2015) by using

95 semantic similarity to find matches between the full set of phenotypes described for a gene and

96 the unique set of phenotypes that characterizes a clade of species, i.e., an 'evolutionary

97 phenotype profile'. The effects from a gene knockdown range from several to hundreds of

98 phenotypes, and the goal is to compare these in their entirety to the calculated set of phenotypes

99 that are variable among the immediate descendants of a particular taxon. Using semantic

100 similarity, the Phenoscape KB performs fuzzy matching between suites of phenotypes, and

101 displays the taxonomic groups that vary in phenotypes that match most closely to the gene

102 profile that results when the action of that gene is disrupted (e.g., knocked down). The user

103 interface provides the statistical support for each match and allows the supporting evidence to be

104 examined. There are some important caveats that must be considered when interpreting the

105 results, such as the potential for some matches to result from differences in annotation coverage

106 between genetic and evolutionary studies in the KB. Potentially spurious matches in that

107 category are flagged by the KB. The KB also provides an interface for the reverse query: what

108 genes have phenotypes that match most closely to the set of evolutionary phenotypes in a 
109 particular taxon under consideration? That is, a biologist who is curious about the genetic basis

110 of taxonomic diversity might want to find genes that have phenotypes that resemble the

111 phenotypic variation exhibited by a particular taxon.

\section{Future applications of semantic similarity to phenotypes of biodiverse taxa}

Questions of whether a particular combination of phenotypes in a taxon is unique, or

114 what it might be similar to, are the types of broad questions that may be addressed in applying

115 semantic similarity-based data mining to phenotypes across diverse taxa. Semantic similarity would retrieve taxa with similar phenotypic profiles; such similarity may have arisen because of

117 common ancestry or independent origin (a 'homoplasy finder'). As described by Braun et al.

118 (Chapter 10), predictive phenomics can, for example, be used to target desired phenotypes in

119 species of interest - and together with recent gene editing capabilities, functional genomic analysis can be newly brought to bear on biodiverse species. The Phenoscape KB currently

121 enables users to view taxa with variation similar to the phenotypic profile of a gene (and vice

122 versa). In the future, they will also be able to query one custom set of phenotypes against another

123 or a taxonomically selected subset, and obtain a ranked list of taxa with similar phenotypes. For

124 example, miniature fishes in the genus Paedocypris, like many fishes that are evolutionarily

125 reduced to an extremely small body size, exhibit the absence of bones including the interhyal,

126 vomer, parietal, posttemporal, and supraneurals (Britz and Conway 2009). Are there other taxa

127 that lack a highly similar set of bones? Enabling a comparison of these phenotypes across

128 diverse taxa would allow a user to query for such matches; in this case, matches would include

129 the ricefishes in the family Adrianichthyidae (Wiley and Johnson 2010), which similarly lack the

130 interhyal, vomer, and supraneurals, and other bones such as the supracleithrum. Further,

131 adrianichthyids may lack or possess extremely small or absent parietal bones and have 
132 structurally simple posttemporal bones, which biologists may recognize as reductive phenotypes

133 on a continuum close to 'absent'. Methods that incorporate a framework of probabilistic

134 reasoning for phenotype relatedness (e.g., Bauer et al. 2012) have the potential to improve

135 precision of ontology-based queries.

\section{Relating biodiverse phenotypes across studies: presence/absence}

Addressing many of the questions in the biodiversity sciences involve knowing how a

specific trait or set of traits has evolved across a group of species. Although the published

literature is replete with research relating species and traits, and a few repositories hold

phylogenetic trees, some of which are computed products from trait data, neither the traits nor

the trees can be easily synthesized across studies. The OntoTrace tool was developed by the

142 Phenoscape team (Balhoff et al. 2014b; Dececchi et al. 2015) to enable users to automatically

143 pull together, from phenotype annotations made to published character matrices and

144 monographic texts (Dececchi et al. 2015, 2016), a set of presence/absence data for specific traits

145 for a set of taxa. For example, querying the Phenoscape KB for a supermatrix of traits of fins,

146 limbs, girdles and their parts in sarcopterygian vertebrates (lobe-finned fishes and tetrapods),

147 Dececchi et al. (2015) retrieved data for 1,052 taxa from 55 studies. The data, 1,759 synthetic

148 presence/absence characters, were derived from 2,588 text-based character states $(1,195$

149 characters). The resultant character by taxon matrix was termed a 'synthetic morphological

150 supermatrix'. Because of the ontological annotations, not only could these phenotypic data be

151 automatically aggregated from multiple studies into a supermatrix, but the asserted data could be

152 extended through inference to traits that were implied by, but not directly asserted in the original

153 publications. For example, if an author observed a curved pectoral fin ray in a species, the

154 machine would infer, based on the knowledge of anatomy encoded in the requisite ontology 
155 (Uberon in this case), that a pectoral fin is present in that species (see Dececchi et al. 2015 and

156 Jackson et al. 2018 for further examples). In this manner, the missing data in the variable

157 character subset of the matrix (the subset containing only characters that include both present and

158 absent states) was reduced from $98.5 \%$ to $78.2 \%$. Further, $76 \%$ of the variable characters were

159 made variable through the addition of inferred states. The authors pointed out that character

160 conflicts and provenance reports from OntoTrace would support researchers review of large

161 aggregated data sets and they showed how such machine reasoning enables quantification and

162 new visualizations of the data, allowing the identification of undersampled character space.

\section{Relating biodiverse phenotypes to phylogenetic trees}

Using ontologies and machine reasoning to automatically generate large, synthetic

character matrices of presence/absence phenotypes (as per above) set the stage for the research of Jackson et al. (2018), who took this a step further. They developed a bioinformatic pipeline to

167 propagate data that was asserted to higher-level taxonomic nodes, to descendant species that

168 were missing data. Similar to Dececchi et al. (2015), they showed that such logic inference

169 significantly extended the asserted data (missing data were reduced from $98.0 \%$ to $85.9 \%$ ), but

170 additionally they showed the value of taxonomic data propagation, which extended the data

171 further, reducing missing data to $34.8 \%$ (Jackson et al. 2018). Using the resultant matrix along

172 with a synthetic phylogeny from the Open Tree of Life (Hinchliff et al. 2015), they mapped the

173 full trait data set for 12,582 species to the tree and addressed the question of how often paired

174 fins were lost in teleost fishes and whether they were ever regained (Jackson et al. 2018).

175 Looking ahead, if all published traits and trees were made computable using these methods, any

176 user could automatically generate a matrix for a specified set of traits and map it on various

177 synthetic tree topologies, which in turn would allow addressing a host of questions regarding the 
178 pattern and tempo of phenotypic evolution and associations with genomic and environmental

179 (Thessen et al. 2015) variables.

\section{Relating biodiverse phenotypes across studies: future work}

As described above, OntoTrace generates synthetic morphological supermatrices for presence/absence characters only (Dececchi et al. 2015). Expanding this functionality to

183 automatically synthesize characters of other qualities, such as shape, size, structure, and color, is

184 a current challenge that the Phenoscape team is addressing. For example, whereas characters in a 185 presence/absence matrix are by definition limited to two states per character, the number of 186 possible states for characters in other categories is a priori unconstrained. Thus, automatically 187 synthesizing characters that, for example, describe 'basihyal bone, shape', can result in a large 188 number of states per character because every originally published state that semantically is some 189 type of 'basihyal bone shape' would have to be appended as a new state to the synthesized 190 character. In the case of this example, there may be seven distinct shape terms used in its 191 annotation (Box 1). The ontological relationships indicate that subsets of these states are more 192 similar to each other than others. By adapting current semantic similarity metrics for the purpose 193 of character and character state aggregation, and in effect, homology assignment, these distinct 194 shape descriptors can be consolidated into new, synthetic states (see matrix in Box 1). 
197 Box 1. Assembling a synthetic character and its states for 'basihyal bone, shape'.

198 Step 1: Assemble list of 'shape' (PATO:0000052) quality terms for all characters and states from 199 multiple publications that include the entity 'basihyal bone' (UBERON:0011618):

200 'spiny' (PATO:0001365)

201 'folded' (PATO:0001910)

202 'upturned' (PATO:0002031)

203 'blade-like' (PATO:0002235)

204 'pointed' (PATO:0002258)

205 'curved ventral' (PATO:0001469)

206 'tapered' (PATO:0001500)

207

208 Step 2: Apply semantic similarity to above list of PATO terms for basihyal bone. Because of 209 higher similarity among terms, three states $(0,1,2)$ are generated from the seven phenotypes:

\section{Character 1: Basihyal bone: shape}

211 Synthetic State 0: 'sharp' (PATO:0001419) (includes 'blade-like', 'pointed', 'tapered')

212 Synthetic State 1: 'curved' (PATO:0000406) (includes 'upturned', 'curved ventral')

213 Synthetic State 2: 'surface feature shape' (PATO:0001925) (includes 'spiny', 'folded')

214

215

216 The Phenoscape team is now developing semantic similarity-based methods to cluster

217 phenotypes across different character categories into characters and states, thus automating 218 matrix construction, and enabling users to optimize the matrix for a variety of metrics. This 
219 would allow a user to constrain the number of characters in a synthesized matrix by excluding

220 those with low information content (e.g., those for high level terms from the anatomy ontology

221 such as 'fin', vs. 'pectoral fin'). Thus, employing semantic reasoning in matrix construction will

222 allow a user to balance the properties of a synthetic matrix between, on the one hand, containing

223 highly specific characters (and thus increased missing data), and on the other, including lower

224 specificity characters (and thus decreasing missing data).

In addition to semantic tools for supermatrix construction, the Phenoscape team is

226 developing enhanced semantics for addressing questions of trait evolution. Unlike the current

227 tools available for analyzing molecular data, where each nucleotide site can be treated as

228 independent of each other, evolutionary models for large morphological character matrices face

229 significant challenges overcoming the strong conditional dependencies and correlations among

230 morphological traits. Most existing methods ignore such dependencies and morphological

231 characters are treated as independent. By leveraging domain knowledge relevant to assessing

232 correlations of the traits underlying the characters, Phenoscape is developing tools that enable

233 users to incorporate evidence of the relatedness of traits in a morphological matrix and into

234 models of character evolution. These include measures of trait independence based on

235 ontological relationships, distance (semantic similarity) of traits in the knowledge graph, and

236 measures of genetic overlap (as derived from gene-phenotype annotations from model organism

237 databases). Such dependencies can be directly built into the macroevolutionary model, or can be

238 used to inform prior probabilities in Bayesian analyses when grouping traits into modules with

239 shared evolutionary parameters or dynamics.

240 One of the challenges in conducting semantic similarity comparisons is the computational

241 overhead of comparing EQ phenotypes over a large ontology space. Improvements in scalability 
242 of semantic similarity methods would enable fast, on-the-fly semantic similarity searches.

243 Successfully applying these methods also currently depends on accurate a posteriori annotation

244 of characters to capture the original author's intent. With only the published description and

245 perhaps images to rely on, curators are unable to consistently apply standardized terms, a factor

246 leading to lower consistency (Cui et al. 2015). For example, in a comparison of curator vs.

247 machine generated annotations (Cui et al. 2015; in prep), three curators described the increased

248 distance between the contralateral pelvic fins with three different quality descriptors: 'far from',

249 'separated from', and 'set apart from'. As methods and software tools develop, such that original

250 authors are better empowered to apply the semantics themselves, the accuracy of character

251 annotation, and thus, consolidation will increase. In the above example, the author would

252 presumably be able to choose, based on the different definitions of the ontology terms, which

253 term is most applicable to the phenotype observed.

\section{Future challenges}

A long-standing question, and one also being currently tackled by the Phenoscape team, is how the relationship of historical homology, i.e., similarity due to common ancestry, can most

257 effectively be used in data retrieval. Recent work by Manda et al. (2016b), examined how 258 semantic similarity is affected when external homology knowledge is included in an ontology.

259 They measured phenotypic similarity between orthologous and non-orthologous gene pairs 260 between humans and either mouse or zebrafish, and they compared the effect of including real 261 vs. faux homology axioms. Semantic similarity was preferentially increased for orthologs when 262 using real homology axioms, though only across the more divergent of the two species (human to 263 zebrash, not human to mouse) (Manda et al., 2016). Overall, the effect of including homology 264 axioms on cross-species semantic similarity was modest, though the authors suggested that the 
effect might be greater for more distant species comparisons. Current efforts include editing and

266 clarifying the homology relationships in the Uberon ontology and investigating how reasoning

267 on different models of homology affects information retrieval in the KB.

268 Another challenge for the broader application of semantics to biodiversity data is the

269 significant, largely manual, effort necessary to annotate phenotypes from the published literature

270 (Dahdul et al. 2015). Natural language processing tools are needed going forward to auto-

271 annotate the legacy literature (Arighi et al. 2013; Cui et al. 2015; in prep). Further, in the future

272 semantic phenotype data may increasingly come directly from publications, as semi-automated

273 methods for marking up manuscripts at the time of publication become more accurate, mature,

274 and thus prevalent. Evaluating, and hence continuously improving the accuracy of machine

275 generated annotations depends on expert-curated "gold standard" data sets. To this end,

276 Phenoscape has developed the first gold standard dataset for biodiversity phenotypes (in prep).

277 Efforts to use ontologies in the process of new species descriptions are underway (Deans et al.

278 2012; Balhoff et al. 2013), and will contribute to achieving a vision of widely available linked

279 species phenotype data.

280 As high-throughput phenotyping, typically involving image data collection, becomes

281 more scalable, the application of semantic metadata would enable automated connections to the

282 tools and computable datasets described herein. These digitization efforts can be new sources of

283 phenotype information (Figure 1). Although broad domains of biology can be served if semantics

284 are placed on digitized images and specimens, so far only a few projects are using semantics to

285 label digitized specimens and their parts, despite promising prototypes (Maglia et al. 2007;

286 Rámirez et al. 2007). If anatomical parts were tagged with ontology terms, then queries on basic

287 trait distributions could be enabled (e.g., presence of pectoral fins in taxa a, b, c...). Although 
288 having a reduced information content compared to full Entity-Quality expressions, entity-only

289 annotations have been shown to be informative for semantic similarity (Manda et al. 2016a).

290 Thus, new sources of phenotypic data, such as those for specimens of extinct and extant taxa

291 associated with institutional collections, can easily be made interoperable through shared

292 semantics (Figure 1).

\section{CONCLUSIONS}

294 Over the past 10 years the development of shared cross-species community ontology resources 295 such as Uberon and PATO has enabled interoperability of phenotype and genotype data. This in

296 turn enables a wealth of potential applications and discoveries from semantic analysis of

297 biodiverse taxa. Scientific attention continues to move toward gaining a deeper fundamental

298 understanding of the developmental and evolutionary relationship between genotype and

299 phenotype. The profound scale and scope of this problem will not only require interoperable big

300 data, both genomic and phenomic, from a biodiverse set of taxa, but also new ways of using

301 machines to enable this understanding. The applications of semantic analysis described herein

302 only scratch the surface of what is possible. As scientific publication moves to incorporate

303 semantic markup of phenotype data, and semi-automated tools are improved to annotate the

304 phenotype legacy literature, knowledge of the rich phenotypic palette of life on our planet can be

305 exposed to machine computation with great advantage to fundamental discovery across the life

306 sciences.

\section{ACKNOWLEDGEMENTS}

308 We thank all participants in the Phenotype Ontology Research Coordination Network (RCN)

309 (NSF 0956049) for their vision, contributions, and commitment to developing shared and 
310 interoperable resources. During the course of this work the Phenoscape project has been

311 supported by NSF awards 1062404, 1062542, 0641025, 1661529, and the National Evolutionary

312 Synthesis Center (NSF 0905606 and 0423641). This manuscript is based in part on work done by

313 P.M.M. while serving at the U.S. National Science Foundation. The views expressed in this

314 paper do not necessarily reflect those of the National Science Foundation or the United States

315 Government.

\section{REFERENCES}

317 Arighi C.N., Carterette B., Cohen K.B., Krallinger M., Wilbur W.J., Fey P., Dodson R., Cooper

318 L., Van Slyke C.E., Dahdul W., Mabee P., Li D., Harris B., Gillespie M., Jimenez S.,

319 Roberts P., Matthews L., Becker K., Drabkin H., Bello S., Licata L., Chatr-aryamontri A.,

320 Schaeffer M.L., Park J., Haendel M., Van Auken K., Li Y., Chan J., Muller H.-M., Cui H.,

321 Balhoff J.P., Chi-Yang Wu J., Lu Z., Wei C.-H., Tudor C.O., Raja K., Subramani S.,

322 Natarajan J., Cejuela J.M., Dubey P., Wu C. 2013. An overview of the BioCreative 2012

$323 \quad$ Workshop Track III: interactive text mining task. Database. 2013:bas056.

324 Balhoff J.P., Dahdul W.M., Dececchi T.A., Lapp H., Mabee P.M., Vision T.J. 2014a. Annotation

325 of phenotypic diversity: decoupling data curation and ontology curation using Phenex. J.

$326 \quad$ Biomed. Semantics. 5:45.

327 Balhoff J.P., Dahdul W.M., Kothari C.R., Lapp H., Lundberg J.G., Mabee P., Midford P.E.,

328 Westerfield M., Vision T.J. 2010. Phenex: ontological annotation of phenotypic diversity.

$329 \quad$ PLoS One. 5(5):e10500.

330 Balhoff J.P., Dececchi T.A., Mabee P.M., Lapp H. 2014b. Presence-absence reasoning for 
evolutionary phenotypes. Peer-reviewed conference paper, Bio-ontologies SIG at ISMB 2014.

Balhoff J.P., Mikó I., Yoder M.J., Mullins P.L., Deans A.R. 2013. A semantic model for species description applied to the ensign wasps (Hymenoptera: Evaniidae) of New Caledonia. Syst. Biol. 62:639-659.

Bauer S., Köhler S., Schulz M.H., Robinson P.N. 2012. Bayesian ontology querying for accurate and noise-tolerant semantic searches. Bioinformatics. 28:2502-2508.

Britz R., Conway K.W. 2009. Osteology of Paedocypris, a miniature and highly developmentally truncated fish (Teleostei: Ostariophysi: Cyprinidae). J. Morphol. 270:389-412.

Cui H., Dahdul W., Dececchi T.A., Ibrahim N., Mabee P., Balhoff J.P., Gopalakrishnan H. 2015. CharaParser+EQ: Performance Evaluation without Gold Standard. Proceedings of the 78th

Cui H., Xu D., Chong S.S., Ramirez M., Rodenhausen T., Macklin J.A., Ludäscher B., Morris Annual Meeting of the Association for Information Science and Technology Annual (ASIS\&T). Vol. 51.

Dahdul W., Dececchi T.A., Ibrahim N., Lapp H., Mabee P. 2015. Moving the mountain: analysis of the effort required to transform comparative anatomy into computable anatomy. Database

Dahdul W.M., Balhoff J.P., Blackburn D.C., Diehl A.D., Haendel M.A., Hall B.K., Lapp H., 
Westerfield M., Mabee P.M. 2012. A unified anatomy ontology of the vertebrate skeletal system. PLoS One. 7(12):e51070.

Dahdul W.M., Balhoff J.P., Engeman J., Grande T., Hilton E.J., Kothari C., Lapp H., Lundberg J.G., Midford P.E., Vision T.J., Westerfield M., Mabee P.M. 2010a. Evolutionary characters, phenotypes and ontologies: curating data from the systematic biology literature.

Dahdul W.M., Cui H., Mabee P.M., Mungall C.J., Osumi-Sutherland D., Walls R.L., Haendel M.A. 2014. Nose to tail, roots to shoots: spatial descriptors for phenotypic diversity in the

Dahdul W.M., Lundberg J.G., Midford P.E., Balhoff J.P., Lapp H., Vision T.J., Haendel M.A., Westerfield M., Mabee P.M. 2010b. The Teleost Anatomy Ontology: anatomical representation for the genomics age. Syst. Biol. 59:369-383.

Deans A.R., Lewis S.E., Huala E., Anzaldo S.S., Ashburner M., Balhoff J.P., Blackburn D.C., Blake J.A., Burleigh J.G., Chanet B., Cooper L.D., Courtot M., Csösz S., Cui H., Dahdul N.M., Friedrich F., Gkoutos G.V., Haendel M., Harmon L.J., Hayamizu T.F., He Y., Hines H.M., Ibrahim N., Jackson L.M., Jaiswal P., James-Zorn C., Köhler S., Lecointre G., Lapp H., Lawrence C.J., Le Novère N., Lundberg J.G., Macklin J., Mast A.R., Midford P.E., Mikó I., Mungall C.J., Oellrich A., Osumi-Sutherland D., Parkinson H., Ramírez M.J., Richter S., Robinson P.N., Ruttenberg A., Schulz K.S., Segerdell E., Seltmann K.C., Sharkey M.J., Smith A.D., Smith B., Specht C.D., Squires R.B., Thacker R.W., Thessen A., Fernandez-Triana J., Vihinen M., Vize P.D., Vogt L., Wall C.E., Walls R.L., Westerfeld M., 
Wharton R.A., Wirkner C.S., Woolley J.B., Yoder M.J., Zorn A.M., Mabee P. 2015. Finding

375 our way through phenotypes. PLoS Biol. 13(1):e1002033.

376

377

378

379

380

381

382

383

384

385

Deans A.R., Yoder M.J., Balhoff J.P. 2012. Time to change how we describe biodiversity. Trends Ecol. Evol. 27:78-84.

Dececchi T.A., Balhoff J.P., Lapp H., Mabee P.M. 2015. Toward synthesizing our knowledge of morphology: using ontologies and machine reasoning to extract presence/absence evolutionary phenotypes across studies. Syst. Biol. 64:936-952.

Dececchi T.A., Mabee P.M., Blackburn D.C. 2016. Data sources for trait databases: comparing the phenomic content of monographs and evolutionary matrices. PLoS One. 11(5):e0155680.

Druzinsky R.E., Balhoff J.P., Crompton A.W., Done J., German R.Z., Haendel M.A., Herrel A., Herring S.W., Lapp H., Mabee P.M., Muller H.-M., Mungall C.J., Sternberg P.W., Van Auken K., Vinyard C.J., Williams S.H., Wall C.E. 2016. Muscle Logic: new knowledge resource for anatomy enables comprehensive searches of the literature on the feeding muscles of mammals. PLoS One. 11(2):e0149102.

Edmunds R.C., Su B., Balhoff J.P., Eames B.F., Dahdul W.M., Lapp H., Lundberg J.G., Vision T.J., Dunham R.A., Mabee P.M., Others. 2016. Phenoscape: identifying candidate genes for evolutionary phenotypes. Mol. Biol. Evol. 33:13-24.

Gkoutos G.V., Green E.C.J., Mallon A.-M., Hancock J.M., Davidson D. 2005. Using ontologies to describe mouse phenotypes. Genome Biol. 6:R8.

Haendel M.A., Balhoff J.P., Bastian F.B., Blackburn D.C., Blake J.A., Bradford Y., Comte A., 
Dahdul W.M., Dececchi T.A., Druzinsky R.E., Hayamizu T.F., Ibrahim N., Lewis S.E.,

Mabee P.M., Niknejad A., Robinson-Rechavi M., Sereno P.C., Mungall C.J. 2014.

Unification of multi-species vertebrate anatomy ontologies for comparative biology in Uberon. J. Biomed. Semantics. 5:21.

Haendel M.A., Neuhaus F., Osumi-Sutherland D., Mabee P.M., Mejino J.L.V., Mungall C.J., Smith B. 2008. CARO--the Common Anatomy Reference Ontology. In: Burger A, Davidson D, Baldock R (eds) Anatomy Ontologies for Bioinformatics. Computational Biology, vol 6. Springer, London.

Hinchliff C.E., Smith S.A., Allman J.F., Burleigh J.G., Chaudhary R., Coghill L.M., Crandall K.A., Deng J., Drew B.T., Gazis R., Gude K., Hibbett D.S., Katz L.A., Laughinghouse H.D. 4th, McTavish E.J., Midford P.E., Owen C.L., Ree R.H., Rees J.A., Soltis D.E., Williams T., life. Proc. Natl. Acad. Sci. U. S. A.

Mabee P., Balhoff J.P., Dahdul W.M., Lapp H., Midford P.E., Vision T.J., Westerfield M. 2012. 500,000 fish phenotypes: The new informatics landscape for evolutionary and developmental biology of the vertebrate skeleton. J. Appl. Ichthyol. 28:300-305.

414 Mabee P.M., Arratia G., Coburn M., Haendel M., Hilton E.J., Lundberg J.G., Mayden R.L., Rios N., Westerfield M. 2007a. Connecting evolutionary morphology to genomics using 
ontologies: a case study from Cypriniformes including zebrafish. J. Exp. Zool. B Mol. Dev. Evol. 308:655-668.

\section{8}

419

420

421

422

423

424

425

426

427

428

429

430

Mabee P.M., Ashburner M., Cronk Q., Gkoutos G.V., Haendel M., Segerdell E., Mungall C., Westerfield M. 2007b. Phenotype ontologies: the bridge between genomics and evolution. Trends Ecol. Evol. 22:345-350.

Maglia A.M., Leopold J.L., Pugener L.A., Gauch S. 2007. An anatomical ontology for amphibians. Proceedings of the Pacific Symposium on Biocomputing 2007:367-378.

Manda P., Balhoff J.P., Lapp H., Mabee P., Vision T.J. 2015. Using the phenoscape knowledgebase to relate genetic perturbations to phenotypic evolution. Genesis. 53:561571.

Manda P., Balhoff J.P., Vision T.J. 2016a. Measuring the importance of annotation granularity to the detection of semantic similarity between phenotype profiles. Proceedings of the International Conference on Biological Ontology 2016.

Manda P., Mungall C.J., Balhoff J.P., Lapp H., Vision T.J. 2016b. Investigating the importance of anatomical homology for cross-species phenotype comparisons using semantic similarity. Proceedings of the Pacific Symposium on Biocomputing 2016.:132-143.

Midford P.E., Dececchi T.A., Balhoff J.P., Dahdul W.M., Ibrahim N., Lapp H., Lundberg J.G., Mabee P.M., Sereno P.C., Westerfield M., Vision T.J., Blackburn D.C. 2013. The Vertebrate Taxonomy Ontology: a framework for reasoning across model organism and species phenotypes. J. Biomed. Semantics. 4:34.

Mullins P.L., Kawada R., Balhoff J.P., Deans A.R. 2012. A revision of Evaniscus (Hymenoptera, 
Evaniidae) using ontology-based semantic phenotype annotation. Zookeys:1-38.

438

439

440

441

442

443

444

445

446

447

448

449

450

451

452

453

454

455

456

457

Mungall C.J., Torniai C., Gkoutos G.V., Lewis S.E., Haendel M.A. 2012. Uberon, an integrative multi-species anatomy ontology. Genome Biol. 13:R5.

Rámirez M.J., Coddington J.A., Maddison W.P., Midford P.E., Prendini L., Miller J., Griswold C.E., Hormiga G., Sierwald P., Scharff N., Benjamin S.P., Wheeler W.C. 2007. Linking of digital images to phylogenetic data matrices using a morphological ontology. Syst. Biol. $56: 283-294$.

Russell J.J., Theriot J.A., Sood P., Marshall W.F., Landweber L.F., Fritz-Laylin L., Polka J.K., Oliferenko S., Gerbich T., Gladfelter A., Umen J., Bezanilla M., Lancaster M.A., He S., Gibson M.C., Goldstein B., Tanaka E.M., Hu C.-K., Brunet A. 2017. Non-model model organisms. BMC Biol. 15:55.

Ruzicka L., Bradford Y.M., Frazer K., Howe D.G., Paddock H., Ramachandran S., Singer A., Toro S., Van Slyke C.E., Eagle A.E., Fashena D., Kalita P., Knight J., Mani P., Martin R., Moxon S.A.T., Pich C., Schaper K., Shao X., Westerfield M. 2015. ZFIN, The zebrafish model organism database: Updates and new directions. Genesis. 53:498-509.

Sprague J., Doerry E., Douglas S., Westerfield M. 2001. The Zebrafish Information Network (ZFIN): a resource for genetic, genomic and developmental research. Nucleic Acids Res. 29:87-90.

The Gene Ontology Consortium. 2017. Expansion of the Gene Ontology knowledgebase and resources. Nucleic Acids Res. 45:D331-D338.

Thessen A.E., Bunker D.E., Buttigieg P.L., Cooper L.D., Dahdul W.M., Domisch S., Franz 
N.M., Jaiswal P., Lawrence-Dill C.J., Midford P.E., Mungall C.J., Ramírez M.J., Specht

459

C.D., Vogt L., Vos R.A., Walls R.L., White J.W., Zhang G., Deans A.R., Huala E., Lewis

460

S.E., Mabee P.M. 2015. Emerging semantics to link phenotype and environment. PeerJ.

461 3:e1470.

462

Vos R.A., Balhoff J.P., Caravas J.A., Holder M.T., Lapp H., Maddison W.P., Midford P.E., Priyam A., Sukumaran J., Xia X., Stoltzfus A. 2012. NeXML: rich, extensible, and verifiable representation of comparative data and metadata. Syst. Biol. 61:675-689.

Washington N.L., Haendel M.A., Mungall C.J., Ashburner M., Westerfield M., Lewis S.E. 2009. Linking human diseases to animal models using ontology-based phenotype annotation. PLoS Biol. 7(11):e1000247.

Wiley E.O., Johnson G.D. 2010. A Teleost Classification Based on Monophyletic Groups. In: Nelson J.S., Schultze H.P., Wilson M.V.H., editors. Origin and Phylogenetic Interrelationships of Teleosts. München, Germany: Verlag Dr. Friedrich Pfeil. p. 123-182.

471 Yoder M.J., Mikó I., Seltmann K.C., Bertone M.A., Deans A.R. 2010. A gross anatomy ontology 472 for hymenoptera. PLoS One. 5(12):e15991. 